\title{
EFFECTS OF ORDER ON SPECTRAL PROPERTIES OF DISCOTIC LIQUID CRYSTAL HEPTYLOXYDIBENZOPYRENE*
}

\author{
P. UZNAŃSKI ${ }^{\dagger}$ \\ Centre of Molecular and Macromolecular Studies of Polish Academy of Sciences \\ Sienkiewicza 112, 90-363 Łódź, Poland
}

(Received July 23, 1998; in final form October 1, 1998)

\begin{abstract}
The spectral properties of discotic heptyloxydibenzopyrene were studied by UV-VIS absorption, fluorescence, and infrared spectroscopy as a function of temperature in two different types of spectroscopic cells. The molecule was found to be a sensitive spectroscopic probe of molecular orientation on a surface. Capillary-filled cells caused the liquid crystal to assume a parallel orientation with respect to the surface, whereas spin-coating caused the orientation of columns to be statistically more non-uniform. It was shown from the spectroscopic studies that the molecular orientation changed on going from crystal to liquid crystalline state and a conclusion was drawn that bulk molecular alignment of columnar phase was defined by an interface imposed by an external surface.
\end{abstract}

PACS numbers: $61.30 . \mathrm{Gd}$

\section{Introduction}

Discotic mesogens forming columnar liquid crystal phases are a new class of organic materials showing fast conduction [1], as well as optoelectronic properties [2], and energy transfer [3], and unique charge transport phenomena [4] along individual molecular columns. In order to understand such diverse phenomena in these systems of low dimensionality, structural information is essential. Especially molecular and macroscopic ordering should have a great effect on further exploration of their intriguing anisotropic physicochemical properties in the neat phases.

Discogenic dibenzopyrenes (DBP) molecules, with a new core fragment of low symmetry $\left(C_{2 h}\right)$, are known to have absorption and fluorescence spectra clearly

*The results, of this paper were initially presented at The Jabtonski Centennial Conference on Luminescence and Photophysics, July 23-27, 1998, Toruń, Poland.

†e-mail: puznansk@bilbo.cbmm.lodz.pl 
distinguishable between a columnar mesophase and a crystalline phase [5]. Thus, their spectroscopic studies can enable a better understanding of an effect of order on the photophysical properties of thin films prepared on external surfaces. Previous studies have shown that the photophysical properties in solution of three DBP derivatives, substituted by eight pentyloxy, heptyloxy or decyloxy substituents, are the same and are not affected by side chain length. Also mesophase absorption and fluorescence spectra are practically the same with a characteristic strong hypsochromic shift for the strongest transition, resulting from exciton coupling between transition moments of the stacked molecules and batochromic shift for weakly allowed transitions. The kinetics of the crystallization process depends on the lateral chain length. For heptyloxy DBP (O7DBP) the initial absorption and fluorescence spectrum of the mesophase, which is supercooled at room temperature, progressively changes its profile as crystallization proceeds, revealing several isosbestic and isoemissive points. This fact allows many experiments to be carried out in order to characterise the structural changes that accompany phase transitions in this liquid crystalline material. In the present study our aim was to test our conclusion concerning the orientation assumed by O7DBP near surfaces, which were based on UV-VIS absorption spectroscopy [5].

The present paper reports how photophysical properties of O7DBP change as a function of thin film order, connected with fabrication method, and as a function of the type of phase. As infrared spectra are highly sensitive to the local structure of liquid crystalline molecules, the problem of molecular ordering in thin films has been studied with a combination of transmission FTIR spectroscopy. It contains: (a) a comparison of the spectroscopic properties of thin films prepared by capillary-filled cells and by spin-coating method; (b) measurements of spectral changes as a function of phase and temperature; (c) the observation that an apparent quantum yield of fluorescence is different for various condensed phases of O7DBP and explanation that it is a function of the macroscopic alignment assumed by the chromophores. This alignment is mainly imposed by the substrate and interface, which restricts liquid crystalline material structure.

\section{Experiment}

1,2,5,6,8,9,12,13-octakis(heptyloxy)dibenzopyrene (Fig. 1) was synthesised as previously described [5]. High-quality thin films for spectroscopic measurements with different macroscopic alignment were prepared either by capillary effect or by a spin-coating method. For the first method spectroscopic cells consisting of two pressed together quartz (UV-VIS) or KRS5 plates (IR) were wedge filled upon heating the powder compound. To achieve a significant molecular orientation, sandwich-like samples were warmed above its isotropic phase for $30 \mathrm{~min}$ and then cooled slowly to room temperature. Spin-coated films of dibenzopyrene were prepared from a single drop of chloroform solution $\left(21 \mathrm{mg} \cdot \mathrm{cm}^{-3}\right)$ onto' a substrate rotating at 1000 r.p.m. The sample alignment was checked using polarizing microscope equipped with a hot stage at $100 \times$ magnification. UV-VIS spectra were recorded using a Specord M40 spectrophotometer. Fluorescence spectra were obtained with SPEX Fluorolog-2 or Perkin-Elmer LS-50 equipped with front face 


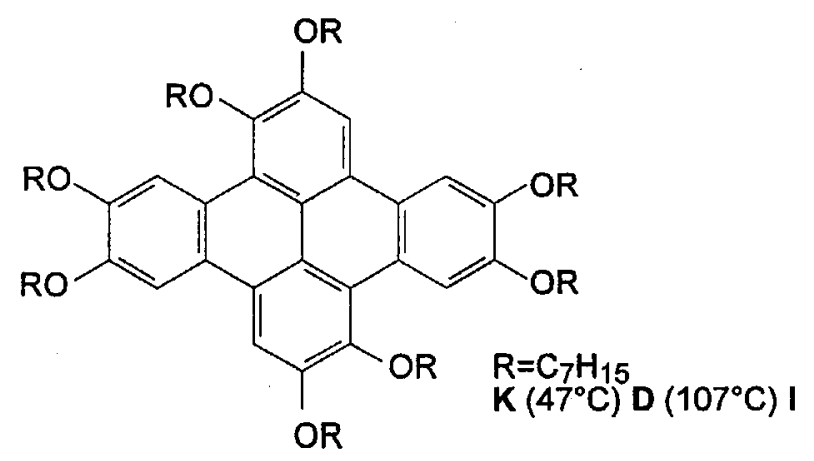

Fig. 1. 1,2,5,6,8,9,12,13-octakis(heptyloxy)dibenzopyrene.

accessory. Circular dichroism spectra were run on a Jobin-Yvone CD-6 spectropolarimeter on thin solution ( $0.01 \mathrm{~mm}$ path length) samples. Infrared spectra were collected with an ATI Mattson Infinity Series FTIR spectrometer. The spectral resolution was maintained at $2 \mathrm{~cm}^{-1}$. The final spectra were averages of 128 interferogram accumulations. During the spectroscopic measurements the temperature of the samples was controlled using holder or cryostat equipped with a PID controller unit.

\section{Results and discussion}

\subsection{Aggregation behaviour of O7DBP in solution}

Although some photophysical electronic spectra in diluted solutions and neat phases of dibenzopyrenes were discussed previously [5], to gain a better understanding of molecular interaction in condensed phases, we have been investigating the relationship between the process of aggregation of O7DBP in apolar solvent and its spectral properties.

The UV-VIS absorption spectra of $n$-heptane solutions containing O7DBP at a concentration above $5 \times 10^{-4} \mathrm{M}$ show a hypsochromic effect (broadening and decrease in intensity) demonstrating a non-Beer's law behaviour. This change in absorption can be correlated with fluorescence and circular dichroism spectra and assigned to an effect of aggregation of O7DBP in non-polar solution. Figure 2 suggests some information on the formation of self-assembled superstructures in $n$-heptane. The fluorescence spectrum in diluted solution of the non-interacting species is very similar to that in crystalline phase. In concentrated solutions the peak at $398 \mathrm{~nm}$ shows an intensity decrease that is attributed to aggregation similar to stacking of the discogens as observed in columnar packing of the mesophase (vide text). A circular dichroism spectrum, obtained for the region where dibenzopyrene core absorbs, provides additional evidence for aggregation. Figure 3 presents the circular and absorption spectra of O7DBP at different concentrations. There is a clear effect of concentration above aggregate threshold formation which appears as a broadening of the spectrum and shape inversion. The CD spectrum 


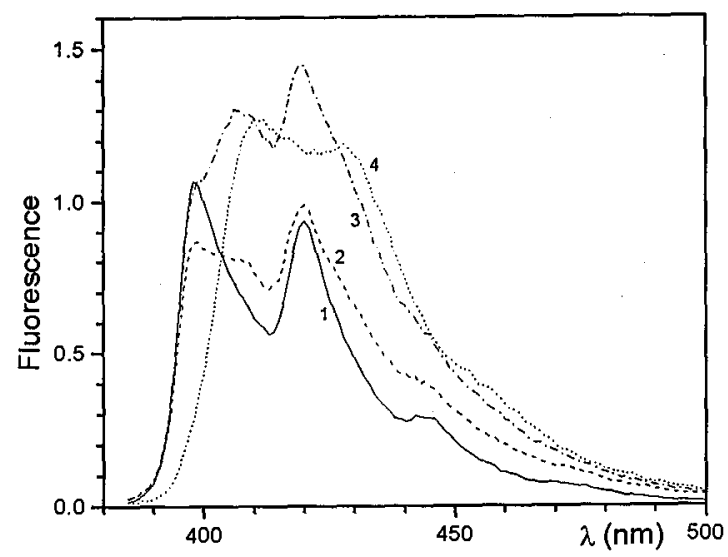

Fig. 2. Fluorescence spectra of O7DBP revealed at different concentrations in $n$-heptane: $1-[\mathrm{c}]=2 \times 10^{-5} \mathrm{M}, 2-[\mathrm{c}]=7 \times 10^{-4} \mathrm{M}, 3-[\mathrm{c}]=10^{-3} \mathrm{M}, 4-$ $[c]=1.5 \times 10^{-2} \mathrm{M}$.

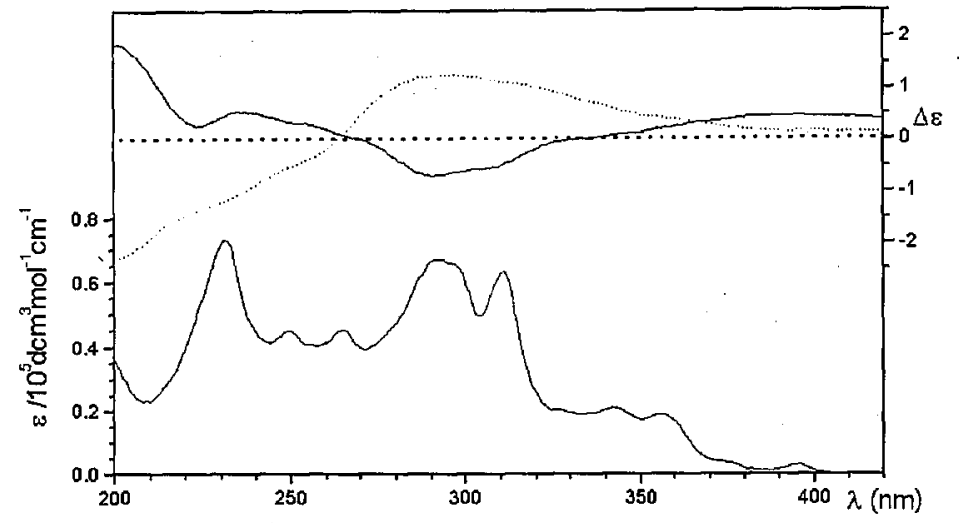

Fig. 3. Absorption and circular dichroism spectra of O7DBP at $2 \times 10^{-5} \mathrm{M}(\longrightarrow)$ and $1.5 \times 10^{-2} \mathrm{M}(\ldots \ldots \ldots)$ in $n$-heptane.

of aggregates has an almost inversed band shape as compared to that observed at low concentration. These changes cannot be unequivocally held responsible for a twisted or random arrangement within the aggregate because in solutions there exist equal populations of right- and left-handed helices [6]: Nevertheless, the results demonstrate that spectral changes observed in non-diluted phases of O7DBP, which will be presented in the next section, are mostly caused by the proximity of the neighbouring chromophores. Specific organization of the chromophores within columns seems to have less effect on fluorescence behaviour.

\subsection{UV-VIS absorption spectra}

Both capillary-filling and spin-coating processes give polycrystalline films, which transmit light between crossed polarizers showing very tiny microcrystals of dimensions of several microns. Heating above $47^{\circ} \mathrm{C}$ results in a complete darkening field of the view for the former sample. In contrast, the latter one exhibits dark 
and bright domains in all orientations relative to the polarizers. This variety is reflected in spectral properties.

Significant differences are found when the spectra obtained for films spin-coated on quartz support are compared with the spectra of the samples between two plates. The absorption spectra of O7DBP thin films depend upon the sample preparation method and hence on the degree of order in the films. Figure 4 shows the absorption spectra of sandwiched samples at various temperatures. As it will become apparent from the later results they represent the most highly ordered films. Curve 1 shows the absorption spectrum of the crystalline phase of O7DBP, curves 2 and 3 discotic phases at $50^{\circ}$ and $100^{\circ} \mathrm{C}$, respectively, while curve 4 the spectrum of O7DBP in its isotropic phase at $115^{\circ} \mathrm{C}$. The intensities of the most strong absorption peaks in discotic and isotropic phases increase relatively to

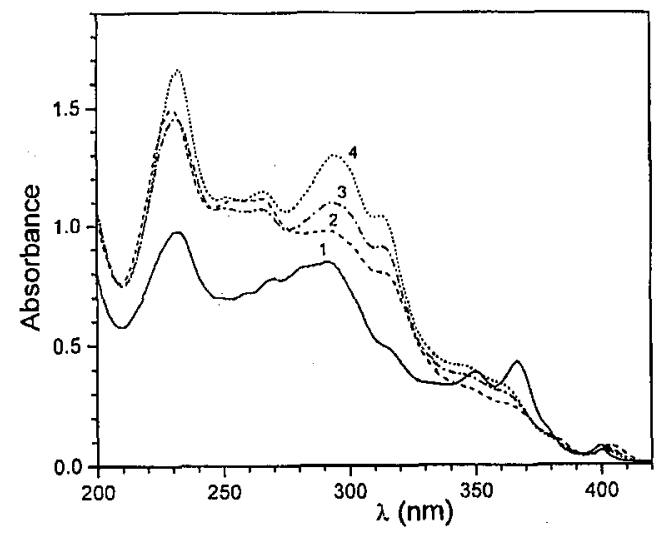

Fig. 4. UV-VIS absorption spectra of O7DBP capillary-filled spectroscopic cell observed for various neat phases: at $45^{\circ} \mathrm{C}(1), 50^{\circ} \mathrm{C}(2), 100^{\circ} \mathrm{C}(3), 115^{\circ} \mathrm{C}$ (4).

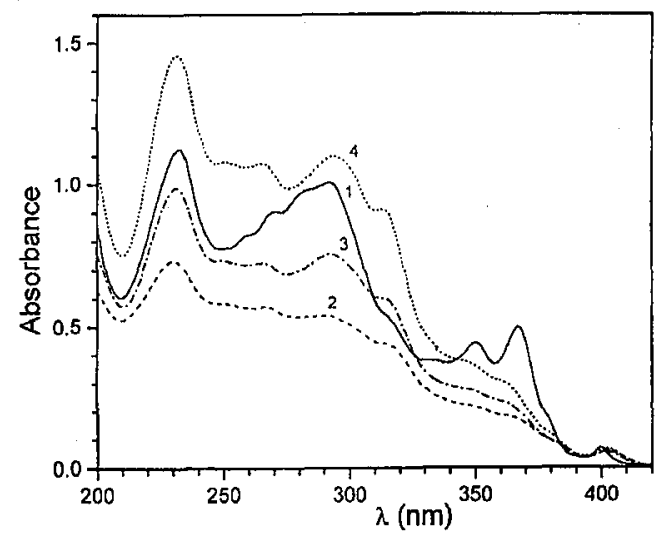

Fig. 5. UV-VIS absorption spectra of O7DBP spin-coated film observed for various neat phases: at $45^{\circ} \mathrm{C}(1), 50^{\circ} \mathrm{C}(2), 100^{\circ} \mathrm{C}(3), 115^{\circ} \mathrm{C}(4)$. 
that observed in crystalline phase, except the well-structured band of medium intensity at $355 \mathrm{~nm}$. The spectral behaviour of spin-coated films, i.e., samples with one free surface (Fig. 5), is different with a temperature change. With increasing temperature the absorbance of the discotic phase (curve 2) first decreases relatively to the started crystalline state, and next it gains intensity. The samples with crystalline and isotropic phase gave essentially the same spectra independent of a sample preparation method. The Stokes shift, which defines the difference between the lowest energy absorption. and emission peaks, does not depend on sample preparation procedure and for crystalline phase it approaches to the value observed for the species in solution. Discotic phase shows always a decrease in Stokes shift with temperature, possibly resulting from a smaller delocalization of the excited state brought about by a weaker intermolecular coupling.

\subsection{Emission properties}

Figure 6 shows the fluorescence spectra made on the same samples as those in Fig. 4. The spectra were excited at the quasi-isoemissive point at $378 \mathrm{~nm}$. Figure 7 collects the integrated emission intensities of these samples as a function of temperature. The fluorescence of a crystalline film has a constant value between $-190^{\circ}$ and $-50^{\circ} \mathrm{C}$, then it slowly increases up to $45^{\circ} \mathrm{C}$. At $50^{\circ} \mathrm{C}$, i.e., slightly above crystal to liquid crystal phase transition, it increases abruptly by a factor of 2.5 . With a further increase in temperature it quickly decreases. Fluorescence spectra of the spun films, with one free surface, are the same as for the sandwiched samples for crystalline and discotic phases. The temperature behaviour is also very similar except the changes of fluorescence around the phase transition temperature at $47^{\circ} \mathrm{C}$ where the emission is indeed at the same intensity.

The results clearly show that the columnar phase emits more efficiently in a sandwiched sample than in a spun one. An essential increase in fluorescence efficiency on going from a solid state to a mesophase is observed. Fluorescence

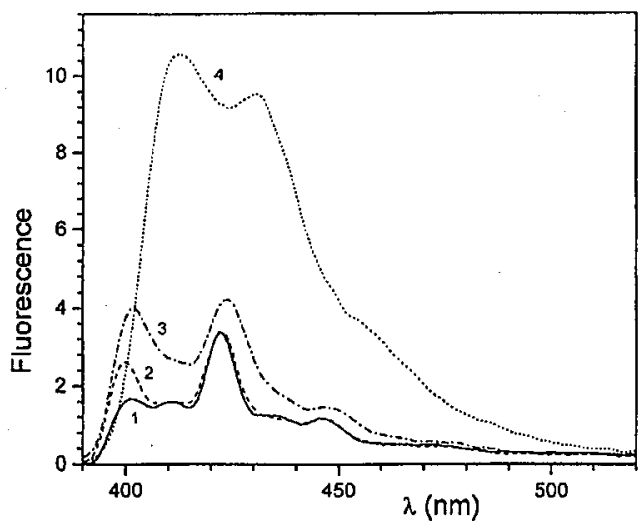

Fig. 6. Changes in fluorescence corrected spectra of O7DBP in capillary-filled cell at different temperatures: at $-190^{\circ} \mathrm{C}(1),-50^{\circ} \mathrm{C}(2), 45^{\circ} \mathrm{C}(3), 50^{\circ} \mathrm{C}(4)$. Spectra were recorded with increasing temperature. 


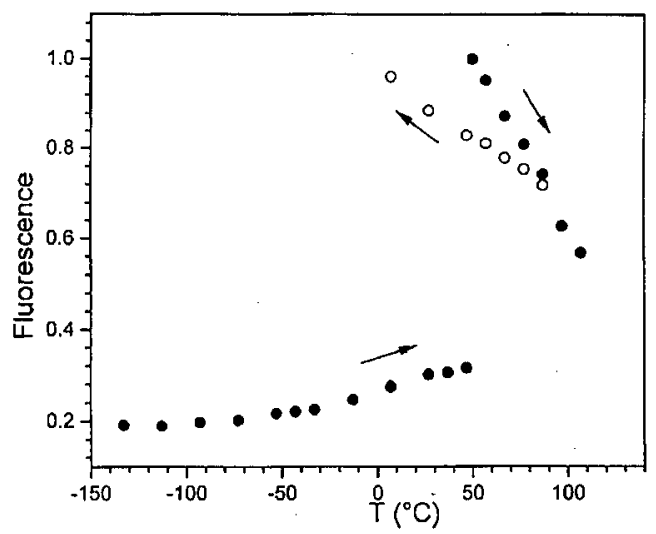

Fig. 7. Variation of the total fluorescence intensity of the spectra presented in Fig. 6 versus temperature upon heating from crystalline phase (full circles) and cooling from isotropic liquid (open circles). Excitation wavelength $\lambda_{\mathrm{ex}}=378 \mathrm{~nm}$. Phase transitions occur at $47^{\circ} \mathrm{C}$ (crystal/liquid crystal) and at $107^{\circ} \mathrm{C}$ (liquid crystal/isotropic liquid).

emission always diminishes with temperature above $47^{\circ} \mathrm{C}$, which can result from enhancement of internal conversion [5]. A rough comparison of the relative quantum yield of emission $(\Phi)$ of the thin films with the known quantum yield in solution measured at the same geometry shows that the crystalline films have the apparent fluorescence efficiency $\left(\Phi^{K}=0.3\right.$ ) comparable to that of the solution species $\left(\Phi^{s}=0.26[5]\right)$. However an increase in fluorescence at phase transition has always been observed for capillary-filled cells $\left(\Phi^{D}=0.75\right)$.

As shown below, also the IR spectra of O7DBP are markedly different for both types of samples.

\subsection{FTIR absorption spectra}

Figures 8 and 9 show infrared spectra of O7DBP in capillary-filled KRS5 cell and spin-coated films supported on one KRS5 substrate, respectively. The vibrations most sensitive to the local structure and molecular ordering are phenyl $\mathrm{C}-\mathrm{H}$ out-of-plane (at $828 \mathrm{~cm}^{-1}$ ) and in-plane (at $1108 \mathrm{~cm}^{-1}$ ) vibrations, $\mathrm{C}-\mathrm{O}$ stretching vibrations in a range of $1200-1300 \mathrm{~cm}^{-1}$ and phenyl $\mathrm{C}-\mathrm{C}$ vibrations in a range of $1350-1500 \mathrm{~cm}^{-1}$. The analysis of vibrations with experimental investigations of the orientation of individual groups with respect to the substrate, by comparing the band intensities, will be published separately [7]. Here we intend to give only a qualitative description of sample alignment, which would complete the photophysical studies.

Remarkable differences are found when infrared spectra of solid phase obtained for cast film on KRS5 are compared with the spectra of the sandwiched samples. These spectroscopic changes can be associated in part with macroscopic orientation of microcrystals, conformation and interaction connected with geometry of a spectroscopic cell [8]. The changes in bands shapes near $1263 \mathrm{~cm}^{-1}$ and $1378 \mathrm{~cm}^{-1}$, which split into several peaks, are the most visible. Although spectra 


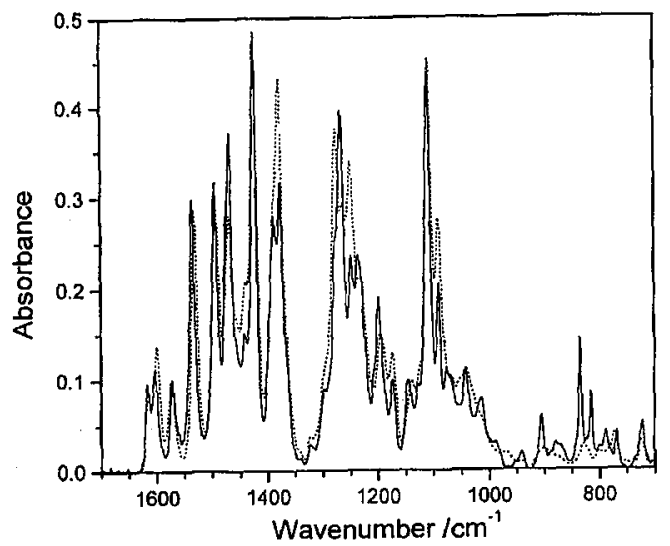

Fig. 8. Infrared spectra of capillary-filled sample taken at $45^{\circ} \mathrm{C}(-)$ and $50^{\circ} \mathrm{C}$ $(\cdots \cdots \cdots)$.

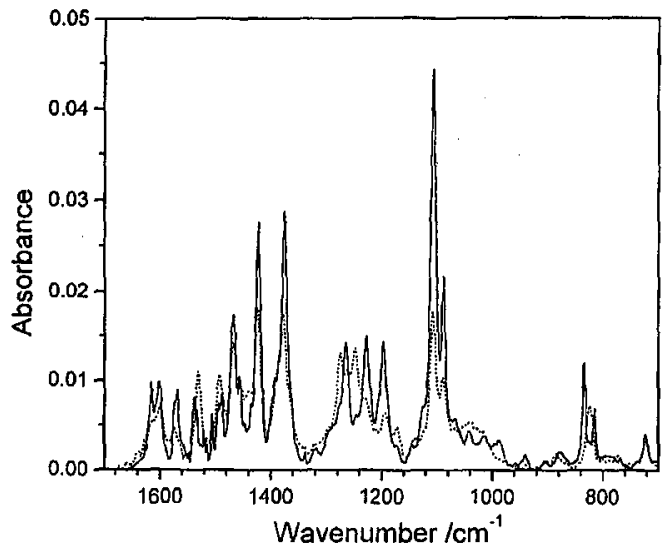

Fig. 9. Infrared spectra of spin-coated sample taken at $45^{\circ} \mathrm{C}(-\longrightarrow)$ and $50^{\circ} \mathrm{C}$ $(\cdots \cdots \cdots)$.

for liquid crystalline phases obtained above the phase transition temperature for these samples are very similar, relative intensities of the bands differ appreciably depending on the fabrication method used. Transition from crystalline to columnar phase for spin-coated samples results in a decrease in the intensity of the in-plane $\phi-\mathrm{H}$ and $\mathrm{C}-\mathrm{C}$ of the benzene ring vibrations indicating an average increase in a tilt angle of dibenzopyrene cores to the surface. However, there are hardly any changes in the intensity of the $\phi-\mathrm{H}$ out-of-plane vibrations. On the contrary, the latter peak for discotic phase observed for capillary-filled sample is of a very low intensity as compared to other samples. The bands near 1108 and $1378 \mathrm{~cm}^{-1}$ also show a marked dependence on sample, being much more intense for the solid state. This corresponds to the changes observed in UV-VIS absorption spectra. On the other hand, at $115^{\circ} \mathrm{C}$, in isotropic phase, the intensity of $\phi$-H out-of-plane band increases supporting homeotropic alignment (aromatic core is parallel to the surface) 
of discotic phase in sandwiched samples. Thus the orientation of O7DBP molecules in the discotic phase can be to some extent controlled by a sample preparation method.

\section{Conclusions}

The changes observed in the UV-VIS absorption and fluorescence spectra of the neat phases of O7DBP correspond to the changes observed in IR region and depend on the type of spectroscopic cell used. These results can be explained taking into account different molecular orientations with respect to the substrate in each phase. The influence of substrate structure on the orientation of the columnar liquid crystals placed between two plates has been recently demonstrated [8]. On glassy surfaces, e.g., triphenylene liquid crystals align homeotropically [9]. Polycrystalline substrate aligns central disks at an angle tilted to the surface [10]. The spectroscopic studies presented here, conducted in non-polarized light confirm that disk-like molecules, display orientational, interactional, and conformational effects depend not only on the structure of a substrate but also on the preparation method. Capillary-filled samples preferentially orient discotic liquid crystals homeotropically with respect to the cell windows, whereas spin-coating of thin films from chloroform solution results in continuous and non-uniform film formation (Fig. 10). This conclusion was drawn from the spectral changes observed in the UV-VIS absorption and fluorescence, and infrared spectra of the neat phases
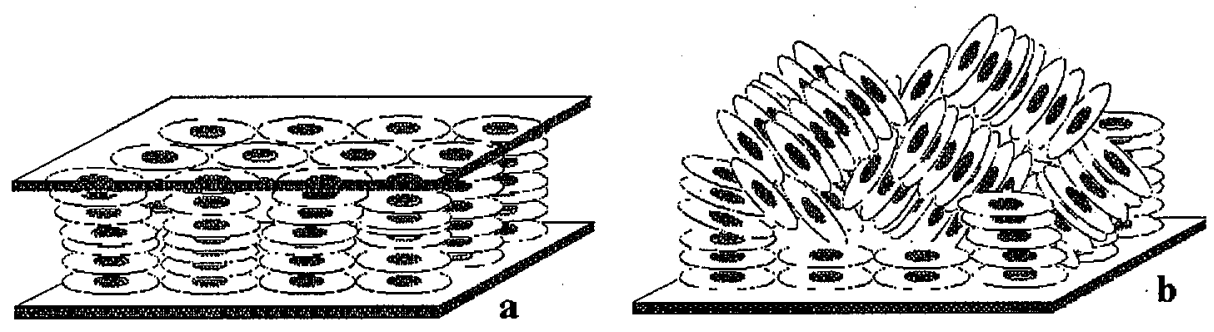

Fig. 10. A schematic illustration of hypothesized alignment of a columnar discotic phase in two different types spectroscopic cells: capillary-filled (a) where the liquid crystal material is pressed between two plates and spin-coated samples (b) supported on one surface. The alignment of the columns within each sample results from different energy surface at the second interfaces in each case.

of O7DBP. The columnar mesophase displays different alignment defined by the interface imposed by the second external surface. Direct structure-spectral property studies, although difficult, are necessary to explain a detailed picture of the molecular alignment in thin films of dibenzopyrene-based liquid crystals.

\section{Acknowledgments}

This work was supported in part by the Committee for Scientific Research (Poland) grant No. 7T08E 06614p04. 


\section{References}

[1] D. Adam, P. Schumacher, J. Simmerer, L. Haussling, K. Siemensmeyer, K.H. Etzbach, H. Ringsdorf, D. Haarer, Nature 371, 141 (1994).

[2] B.A. Gregg, M.A. Fox, A.J. Bard, J. Phys. Chem. 93, 4227 (1989).

[3] D. Markovitsi, A. Germain, P. Millie, I. Lécuyer, L. Gallos, P. Argyrakis, H. Bengs, H. Ringsdorf, J. Phys. Chem. 99, 1005 (1995).

[4] N. Boden, R.J. Bushby, J. Clements, B. Movaghar, J. Appl. Phys. 83, 3207 (1998).

[5] P. Uznański, S. Marquet, D. Markovitsi, P. Schumacher, H. Ringsdorf, Mol. Cryst. Liq. Cryst. 293, 123 (1997).

[6] J.P. Gallivan, G.B. Schuster, J. Org. Chem. 60, 2423 (1995).

[7] P. Uznański, in preparation.

[8] X. Yang, S.A. Nitzsche, S.L. Hsu, D. Collard, R. Thakur, C.P. Lillya, H.D. Stidham, Macromol. 22, 2611 (1989).

[9] G. Kruk, A. Kocot, R. Wrzalik, J.K. Vij, O. Karthaus, H. Ringsdorf, Liq. Cryst. 14, 807 (1993).

[10] T.S. Perova, J.K. Vij, Adv. Mater. 7, 919 (1995). 